\title{
LEFT ATRIAL MYXOMA CAUSING OPHTHALMIC ARTERY OCCLUSION
}

\author{
P. E. RAFUSE, D. A. NICOLLE, C. M. L. HUTNIK and C. E. PRINGLE \\ London, Ontario
}

\begin{abstract}
SUMMARY
A 45-year-old Caucasian woman presented with sudden onset right-sided hemiparesis, aphasia and a painful left eye. Examination revealed a bone-white fundus with no perfusion of either the retinal or choroidal circulations. Magnetic resonance imaging showed increased signal density of the left optic nerve sheath, orbital fat and extraocular muscles consistent with infarction of the ophthalmic artery distribution. An echocardiogram disclosed a mobile, multilobulated mass attached to the septal wall of the left atrium. Pathological examination of the resected tumour confirmed the diagnosis of endocardial myxoma. A colour Doppler study performed 1 month after surgery demonstrated absence of flow in the left ophthalmic artery. At 2 months, the left eye had no light perception and an intraocular pressure of $2 \mathrm{mmHg}$. This clinicopathological report describes the rare presentation of an acute ophthalmic artery obstruction secondary to atrial myxoma.
\end{abstract}

Acute ophthalmic artery occlusion is an infrequent event. Vascular occlusions in the eye more commonly involve either the retinal or choroidal circulations. In one survey of arterial occlusions of the eye, acute ophthalmic artery occlusion accounted for only 1 of 62 cases. $^{1}$ This clinicopathological report describes a patient with an acute ophthalmic artery occlusion due to an atrial myxoma. This diagnosis was supported by magnetic resonance imaging (MRI) of the head and orbits, intravenous fluorescein angiography of the retina and choroid, colour Doppler imaging, echocardiography and pathological examination of an endocardial tumour excised from the left atrium.

From: Department of Ophthalmology and Clinical Neurological Sciences, University of Western Ontario, London, Ontario, Canada.

Correspondence to: Dr David A. Nicolle, University Hospital, Department of Ophthalmology, 339 Windermere Road, London, Ontario, Canada N6A 5A5.

\section{CASE REPORT}

A 45-year-old Caucasian woman was found collapsed on the floor of her home. In addition to a right hemiparesis and global aphasia, examination revealed several ocular findings. The eyes were tonically directed in left gaze but full horizontal excursions were noted bilaterally on oculocephalic testing. A right upper motor neuron seventh cranial nerve palsy was present. There was marked conjunctival injection of the left eye. The left pupil was eccentric, mid-dilated and a left afferent pupillary defect was noted. Ophthalmoscopy of the left eye revealed a bone-white fundus with no apparent perfusion of either the retinal or choroidal circulations. There was some residual blood in the retinal veins and "box car" segmentation of the blood column in several mid-peripheral arterioles. The optic disc appeared pale and swollen.

Five days after presentation, the left fundus displayed retinal and pre-retinal haemorrhages (Fig. 1a). The fundus of the right eye was normal (Fig. 1b) compared with the left eye at 24 days post-occlusion (Fig. 1c), the latter showing substantial clearing of the haemorrhage. Follow-up photographs taken at 2 months (Fig. 1d) and 7 months (Fig. 1e) showed progressive fibrosis of the optic disc and retina. An intravenous fluorescein angiogram, performed 24 days after occlusion, showed abnormal delayed filling of the choroid in conjunction with no filling of the central retinal artery of the left eye (Fig. 2a). Later frames showed a hyperfluorescent choroid with some blockage from the retinal haemorrhage in the left eye compared with the normal appearance of the right eye (Fig. 2b-d).

Computed tomography without contrast ruled out intracranial haemorrhage. MRI revealed multiple areas of signal changes consistent with old and new infarcts. There appeared to be acute involvement of the posterior left internal capsule, basal ganglion and optic nerve sheath. The fat and extraocular muscles

Eye (1997) 11, 25-29 사 1997 Royal College of Ophthalmologists 


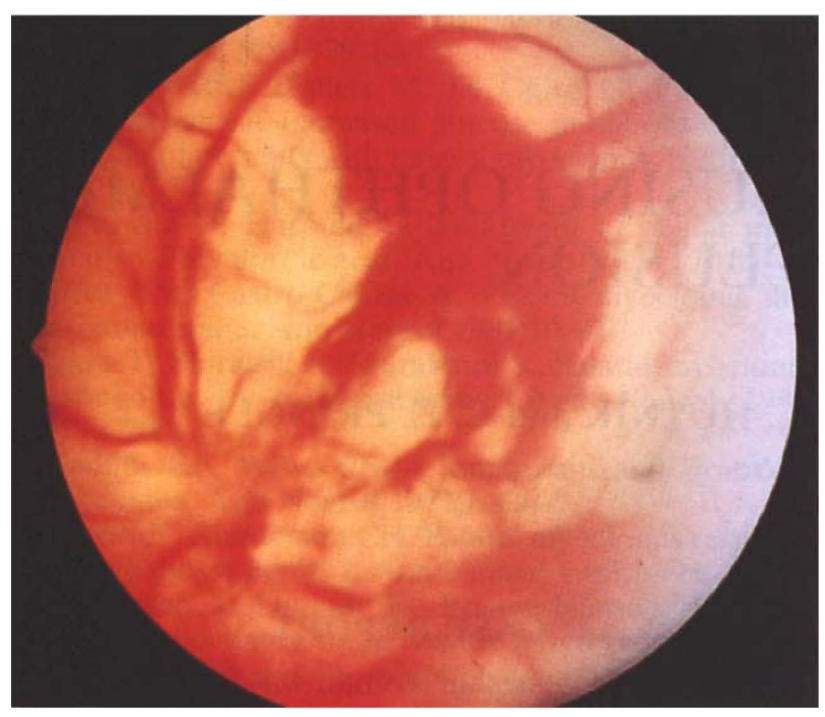

(a)

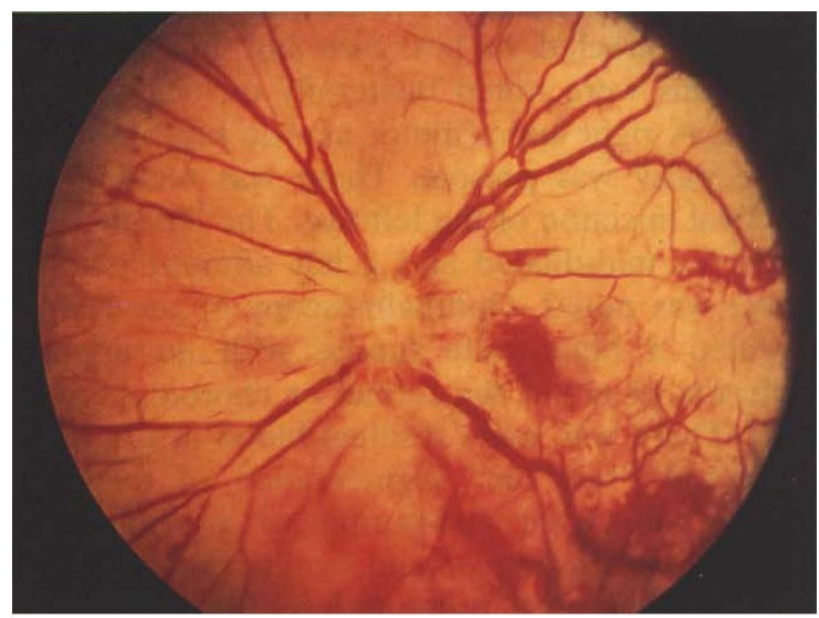

(c)

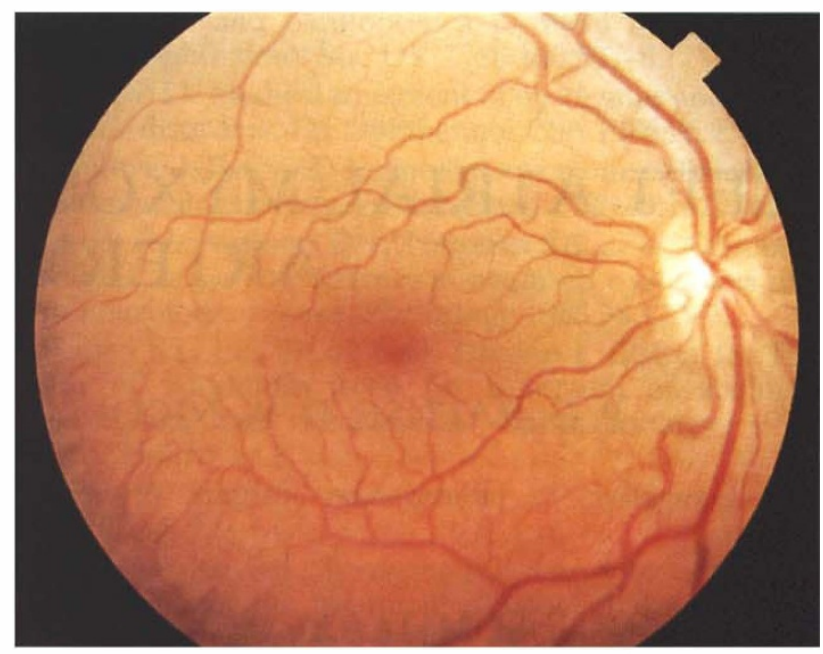

(b)

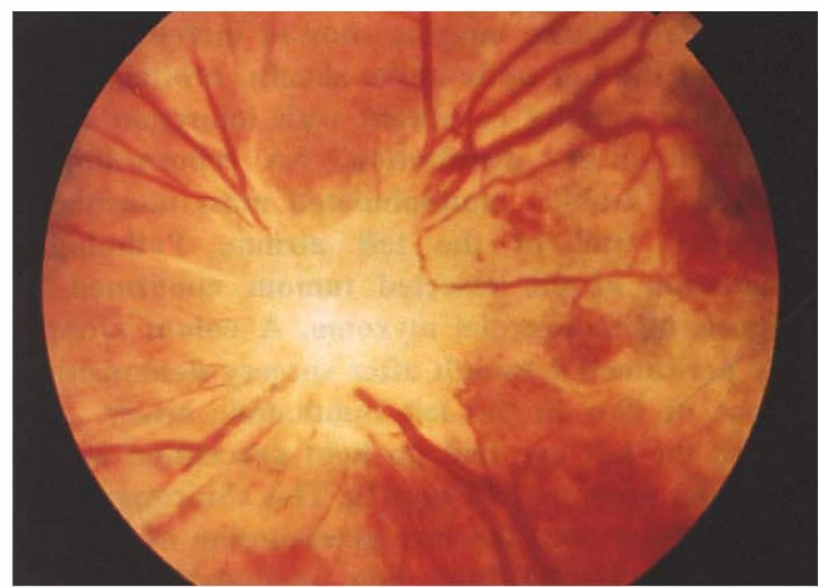

(d)

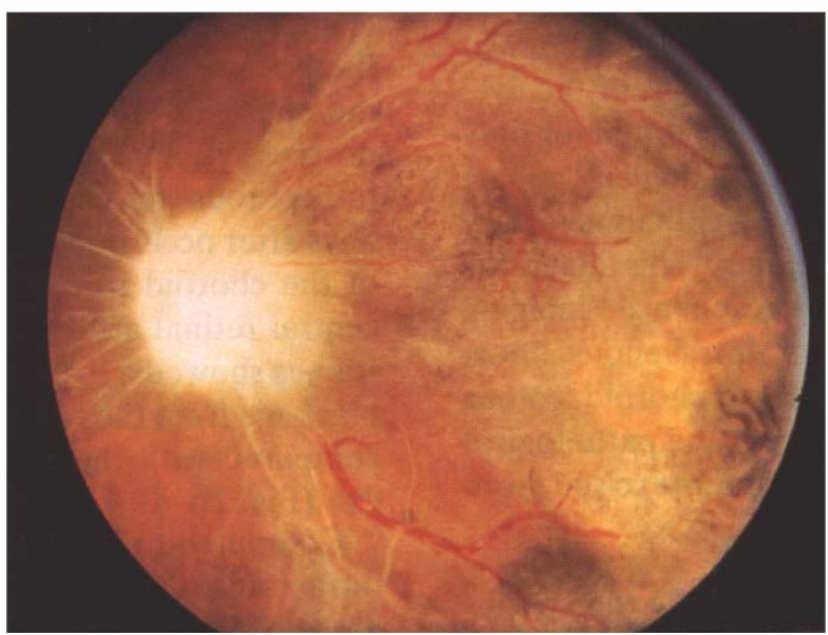

(e)

Fig. 1. Colour fundus photographs of: (a) left eye, taken in the Intensive Care Unit with a Kowa camera 5 days after ophthalmic artery occlusion; (b) right eye, 24 days post-occlusion; (c) left eye, 24 days post-occlusion; (d) left eye, 2 months after occlusion; (e) left eye, 7 months after occlusion. (b)-(e) were taken with a Zeiss fundus camera. 


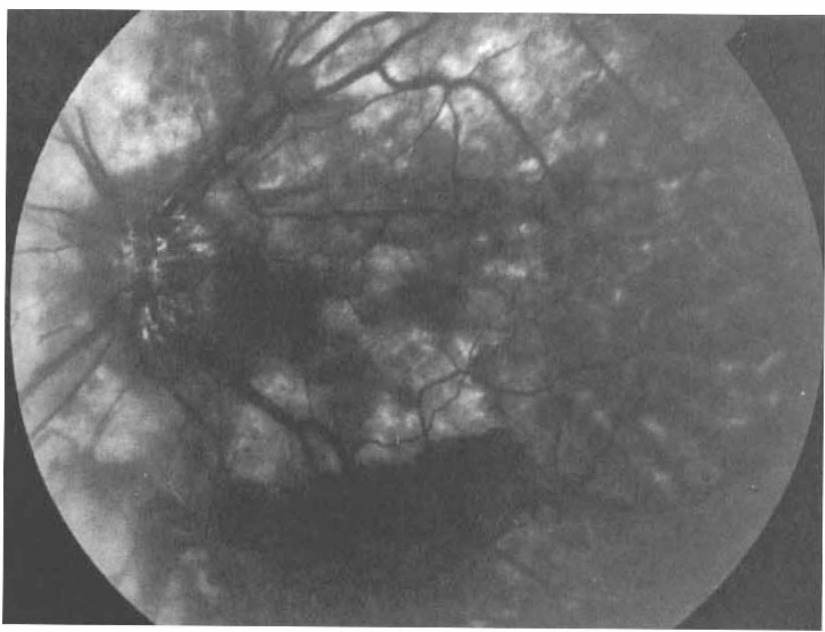

(a)

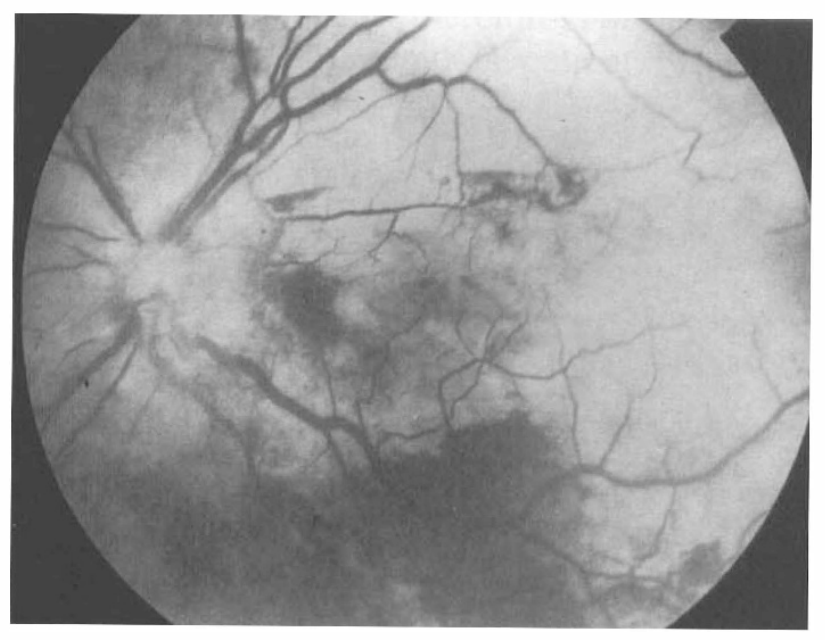

(c)

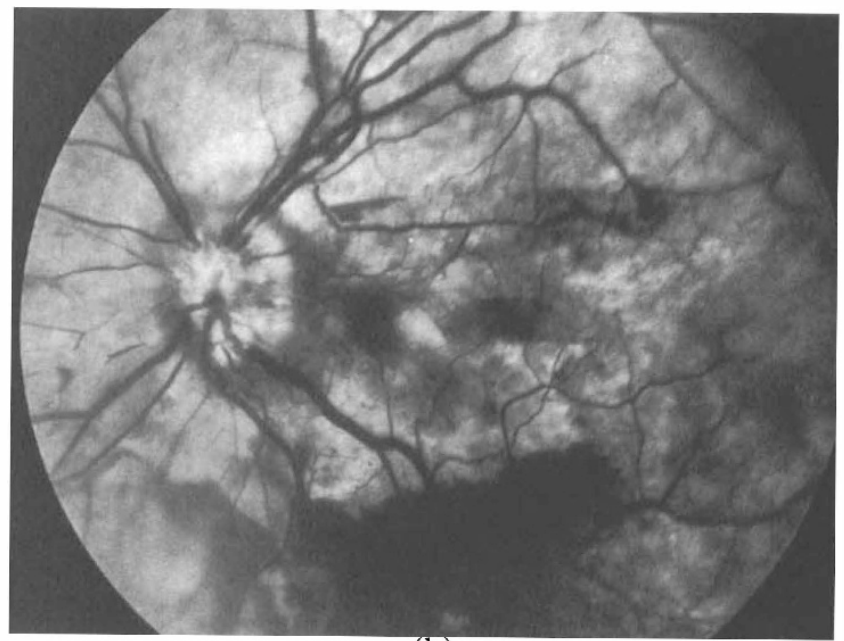

(b)

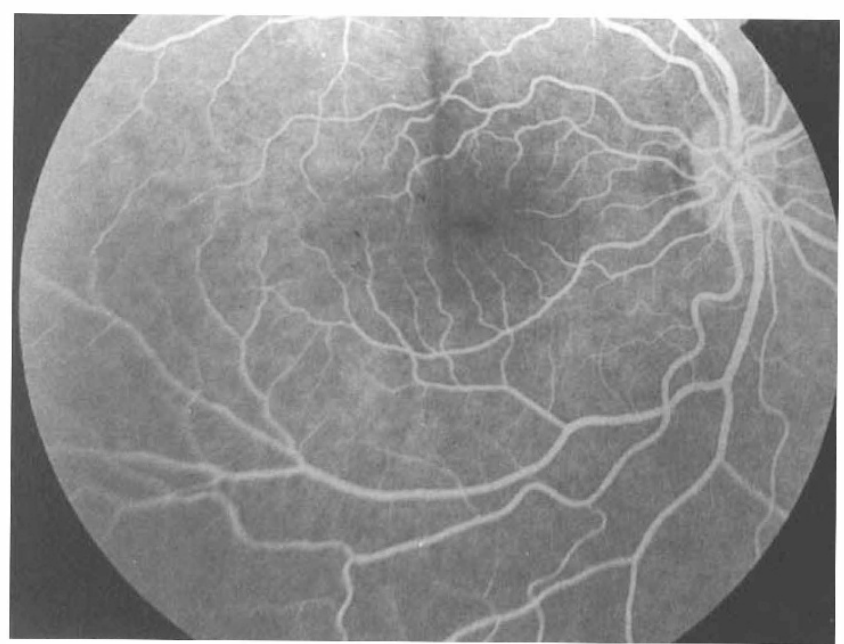

(d)

Fig. 2. Fluorescein angiograms taken 24 days post-occlusion of: (a) left eye at 12 seconds showing no choroidal filling; (b) left eye at 55 seconds showing no filling of the retinal vessels but increased hyperfluorescence of the choroid; (c) left eye at 973 seconds showing marked staining of the choroid; (d) right eye at 116 seconds showing normal fluorescein pattern.

of the left orbit showed increased signal intensity suggesting acute infarction.

An echocardiogram revealed a large, mobile, multilobular mass arising from the left atrium. It prolapsed into the left ventricle during diastole (Fig. 3a,b). Pathological examination of the resected mass confirmed the diagnosis of atrial myxoma (Fig. 4a,b).

Two months after presentation the left eye was comfortable with no light perception and a full left afferent pupillary defect. Intraocular pressure was $2 \mathrm{mmHg}$. There was no evidence of neovascularisation of the anterior or posterior segment. The fundus was pale and there was clearing of retinal haemorrhages. The disc was pale and atrophic.

A colour Doppler imaging study performed 3 months after initial presentation showed markedly diminished flow in the left ophthalmic artery compared with the right, with some perfusion of the posterior ciliary circulation.

\section{DISCUSSION}

Acute obstruction of the ophthalmic artery is an uncommon event. One survey of 8 cases found a variety of causes including carotid artery disease, orbital mucormycosis, atrial fibrillation, external compression of the globe and orbit, trauma, and one case of embolism from an atrial myxoma. ${ }^{2}$ The last case, in a 16-year-old male, represents the only other reported instance of this tumour causing acute ophthalmic artery obstruction.

Atrial myxomas more frequently cause retinal embolism. Manschot ${ }^{3}$ initially recognised and reported an association of central retinal artery embolism with an endocardial myxoma. Subsequently, atrial myxomas have been implicated in the pathogenesis of both isolated retinal and choroidal infarctions. $^{4-7}$

Table I summarises a number of clinical and ophthalmic investigations that can aid in differentiat- 


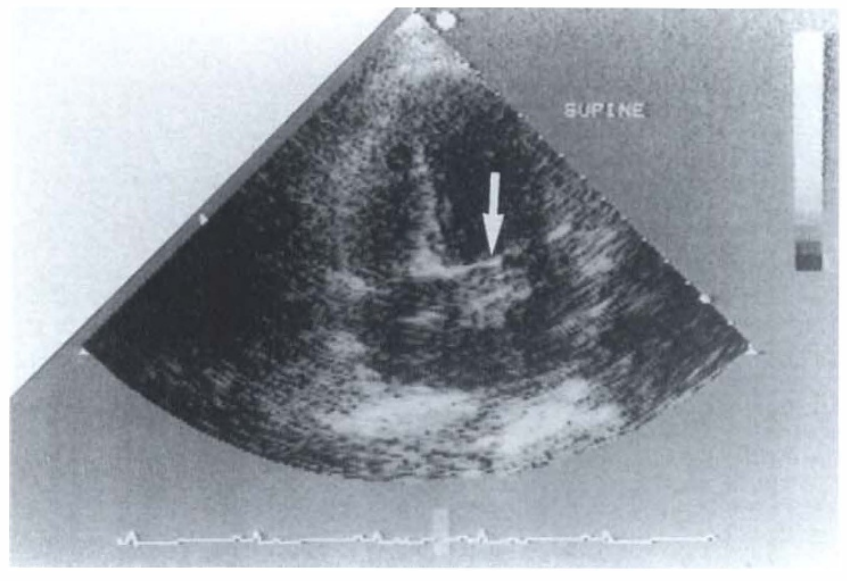

(a)

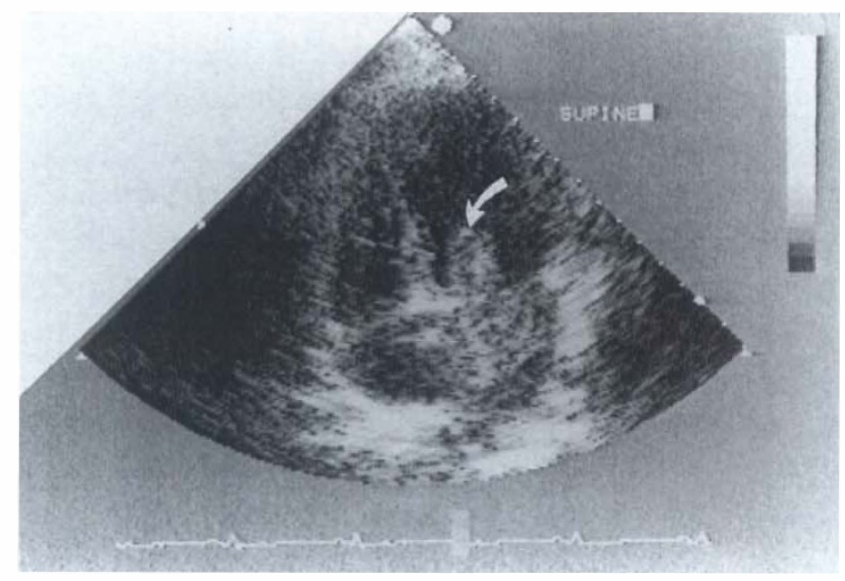

(b)

Fig. 3. Echocardiogram showing atrial mass during (a) systole (straight arrow); and (b) diastole, showing prolapse of the tumour into the left ventricle (curved arrow).

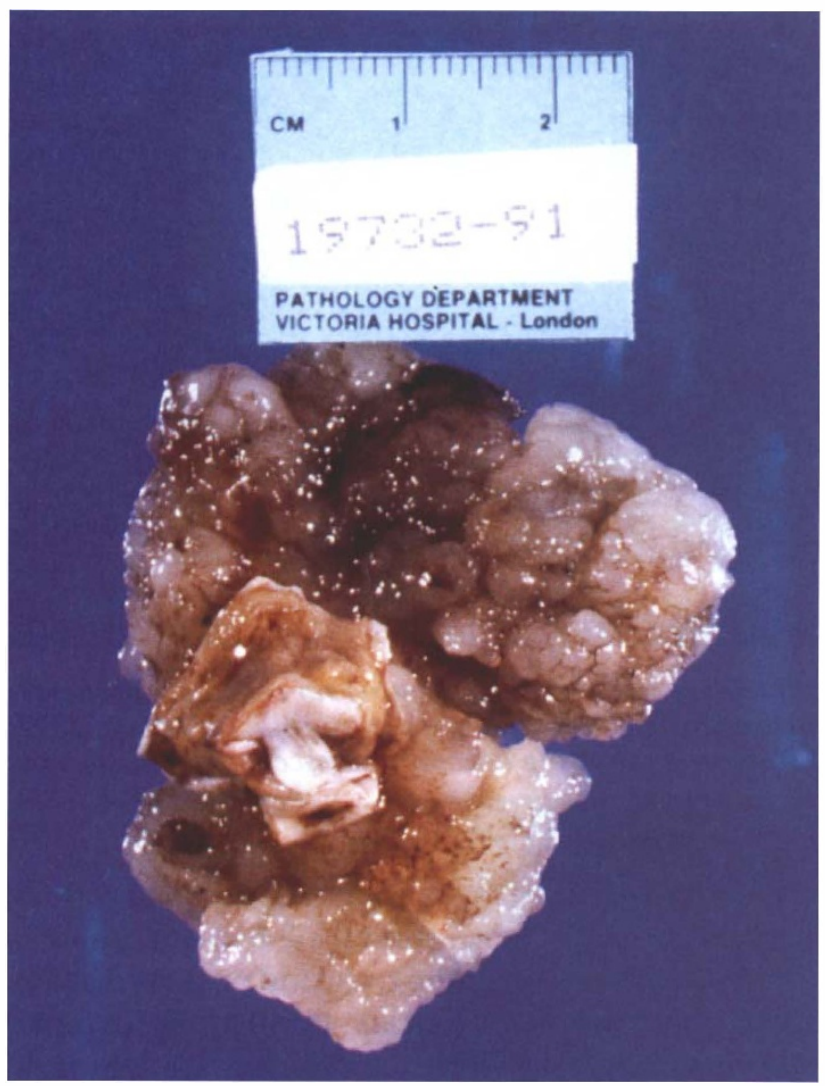

(a)

ing obstruction of the central retinal artery from ophthalmic artery obstruction. The pain associated with the latter arises from the rich sensory innervation of the ischaemic choroid. The visual prognosis is invariably worse. ${ }^{2,8}$ The associated ischaemia of the ciliary body results in decreased aqueous production and prolonged hypotony. ${ }^{9}$ Infarction of the choroid precludes observation of a cherry-red spot. Fluorescein angiography reveals choroidal flow abnormalities characterised by late

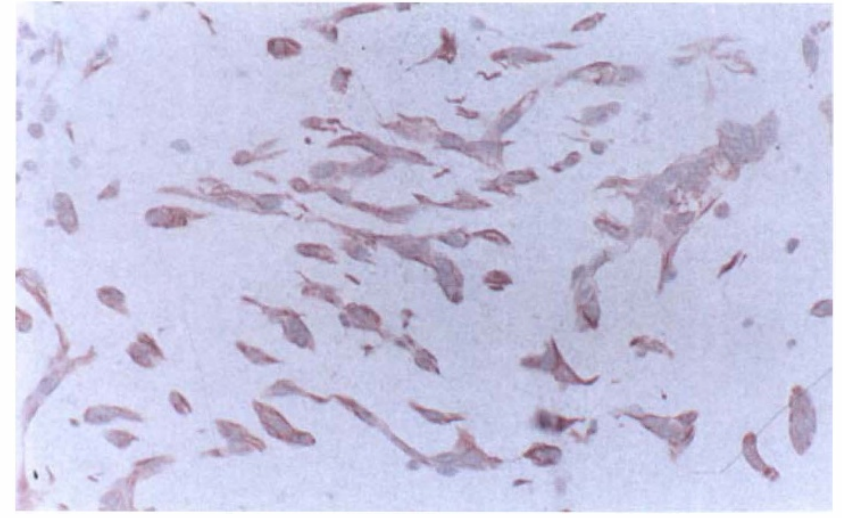

(b)

Fig. 4. Pathology of resected left atrial mass: (a) gross and (b) microscopic using Movat's stain. The stroma is filled with polygonal and stellate cells surrounded by abundant, loose mucoid material characteristic of an atrial myxoma.

leakage ascribed to the ischaemic retinal pigment epithelium becoming incompetent and unable to contribute to maintenance of the blood-retinal barrier.

Confirmation of simultaneous retinal, choroidal and orbital infarction can be obtained with MRI and colour Doppler imaging. With this latter technique, singular occlusion of the ophthalmic artery can be distinguished from two or more obstructions of more terminal retinal and choroidal branches. ${ }^{10}$ 
Table I. Clinical features and investigations for differentiating central retinal artery obstruction (CRAO) from ophthalmic artery obstruction (OAO)

\begin{tabular}{lll}
\hline Features & CRAO & OAO \\
\hline Pain and tenderness & None & Present \\
Visual acuity & CF to HM & Usually NLP \\
Fundus appearance & \pm cherry-red spot & Non-perfused fundus \\
IOP & Normal & Decreased \\
IVFA & Decreased retinal flow & $\begin{array}{l}\text { Decreased retinal and choroidal flow with late } \\
\text { choroidal stain }\end{array}$ \\
\hline
\end{tabular}

$\overline{\mathrm{CF}}$, counting fingers; HM, hand movement; NLP, no light perception; IOP, intraocular pressure; IVFA, intravenous fluorescein angiography.

In contrast to the ocular ischaemic syndrome seen in chronic carotid artery disease, 11 the abrupt cessation of blood flow to the orbit does not seem to be associated with neovascularisation of the anterior segment and development of glaucoma. ${ }^{2}$ Follow-up after a sudden blockage of ophthalmic artery blood flow may, therefore, not need to be as frequent as that required in patients with carotid artery disease and ocular ischaemic syndrome.

Key words: Atrial myxoma, Opthalmic artery obstruction.

\section{REFERENCES}

1. Henkind P. Symposium: Retinal vascular disease. Introduction and phenomenology. Trans Am Acad Ophthalmol Otolaryngol 1977;83:OP367-72.

2. Brown GC, Magargal LE, Sergott R. Acute obstruction of the retinal and choroidal circulations. Ophthalmology 1986;93:1373-82.

3. Manschot WA. Embolism of the central retinal artery originating from an endocardial myxoma. Am J Ophthalmol 1959;48:381-5.
4. Anderson JD, Lubow M. Atrial myxoma as a source of retinal embolisation. Am J Ophthalmol 1973;76: 769-72.

5. Cogan DG, Wray SH. Vascular occlusions in the eye from cardiac myxomas. Am J Ophthalmol 1975; 80:396-403.

6. Jampol LM, Wong AS, Albert DM. Atrial myxoma and central retinal artery occlusion. Am J Ophthalmol 1973;75:242-9.

7. Yasuma F, Tsuzuki M, Yasuma T. Retinal embolism from left atrial myxoma. Jpn Heart J 1989;30:527-32.

8. Brown GC, Margaral LE. Central retinal artery obstruction and visual acuity. Ophthalmology 1982; 89:14-9.

9. Pederson JE. Hypotony. In: Tasman W, Jaeger EA, editors. Duane's clinical ophthalmology, vol 3. Philadelphia: JB Lippincott, 1984:58:1-8.

10. Lieb WE, Cohen SM, Merton DA, et al. Colour Doppler imaging of the eye and orbit: technique and normal vascular anatomy. Arch Ophthalmol 1991; 109:527-31.

11. Bullock JD, Falter RT, Downing JE, Snyder HE. Ischaemic ophthalmia secondary to an ophthalmic artery occlusion. Am J Ophthalmol 1972;74:486-93. 